UOT 547.97+535.37

\title{
RESEARCH INTO ONE-STEP THREE COMPONENT REACTION OF SOME YLIDENECYANOACETAMIDES (OR YLIDENEMALONONITRILES), MALONONITRILE AND 1,3-DIAMINOPROPANE
}

\author{
F.N. Naghiyev \\ Baku State University, Chemical Faculty \\ Z. Khalilov 23, Baku, Azerbaijan, e-mail: farid.orgchemist@gmail.com
}

Received 21.04.2019

\begin{abstract}
The one-step interaction of substituted ylidenecyanoacetamides (or ylidenemalononitriles), malononitrile and 1,3-diaminopropane was carried out in methanol environment at room temperature and it established the formation of new substituted dihydropyridopyrimidine derivatives. It also revealed that under the same conditions through the use of one-step threecomponent reaction of pyridylidenecyanoacetamide (or pyridylidenemalononitrile), 2-chloro-5nitrobenzylidenecyanoacetamide (or 2-chloro-5-nitrobenzylidenemalononitrile), 2,6dichlorobenzylidenecyanoacetamide (or 2,6-dichlorobenzylidenemalononitrile), malononitrile and 1,3-diaminopropane there formed substituted derivatives not of dihydropyridopyrimidine but tetrahydropyridopyrimidine. Structures of all synthesized compounds were verified by NMR and $X$-Ray spectroscopy.

Keywords: ylidenecyanoacetamides, pyridylidenecyanoacetamide, malononitrile,

1,3-diaminopropane, $N M R$
\end{abstract}

DOI: $10.32737 / 2221-8688-2019-2-275-281$

\section{Introduction}

The literature refers to new methods of theirv ibhibiting properties in respect of production of pyrimidine derivatives and various disease-producing factors [1-12].

\section{Results and discussion}

We carried out the one-step three at room temperature, for 24-28 hours and component interaction of various established the possibility of production of ylidenecyanoacetamides (or ylidenema- substituted new dihydropyrido[1,2lononitriles), malononitrile and 1,3- $a$ ]pyrimidines (4a-j) irrespective of the nature diaminopropane in methanol environment and of polarized double bond.

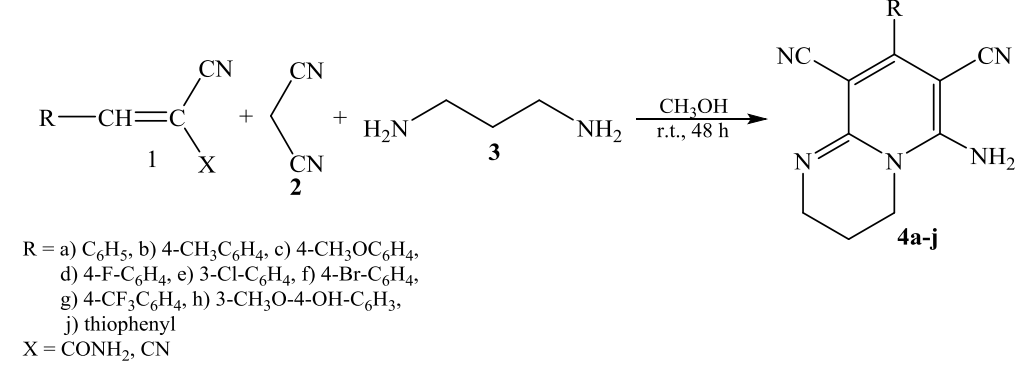

A plausible mechanism of the reaction is presented below: 
$(\mathrm{NC})_{2} \mathrm{CH}_{2}+\mathrm{H}_{2} \mathrm{~N}\left(\mathrm{CH}_{2}\right)_{3} \mathrm{NH}_{2} \rightleftharpoons(\mathrm{NC})_{2} \stackrel{\overline{\mathrm{C}}}{\mathrm{H}}+\stackrel{\mathrm{H}_{3}}{\mathrm{~N}}\left(\mathrm{CH}_{2}\right)_{3} \mathrm{NH}_{2}$

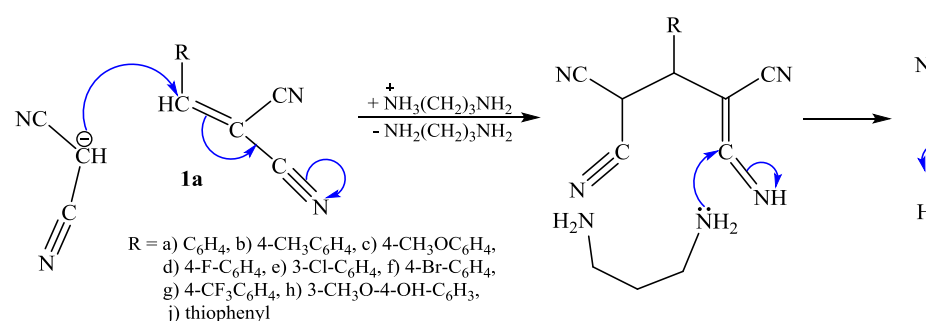<smiles>[R]C(C(C#N)=C(N)NCCCN)C([R])C(C#N)=C(N)C(=N)NCCCN</smiles><smiles>[R]C1CC(CC)(CN)N(CCCN)C(N)=C1C#N</smiles><smiles>[R]C1CCC2NCCCN2C(N)=C1C#N</smiles><smiles>[R]C1=C(C#N)C2NCCCN2C(N)=C1C</smiles><smiles>[R]C1=NC2=NCC[C@@H](C#N)N2C(N)=C1C#N</smiles>

Through the use of similar one-step threecomponent reaction of pyridylidene cyano acetamide (or pyridylidenemalononitrile) as compound with polarized double-bond,2,6dichlorobenzylidenecyanoacetamide (or 2,6dichlorobenzylidene malononitrile),

malononitrile and 1,3-diaminopropane at the same conditions, it became possible to establish the formation of corresponding substituted tetrahydropyrido[1,2-a]pyrimidine derivatives $(\mathbf{6}, \mathbf{8}, \mathbf{1 0})$, not dihydropyrido[1,2-a]pyrimidines.

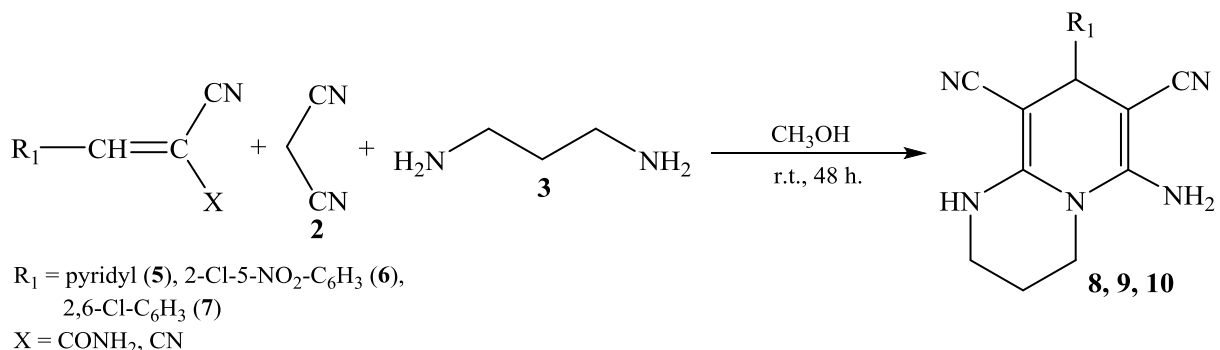

A plausible reaction mechanism and ${ }^{1} \mathrm{H},{ }^{13} \mathrm{C}$ NMR spectra (fig.1, fig.2) of compound $4 \mathbf{e}$ is presented below.
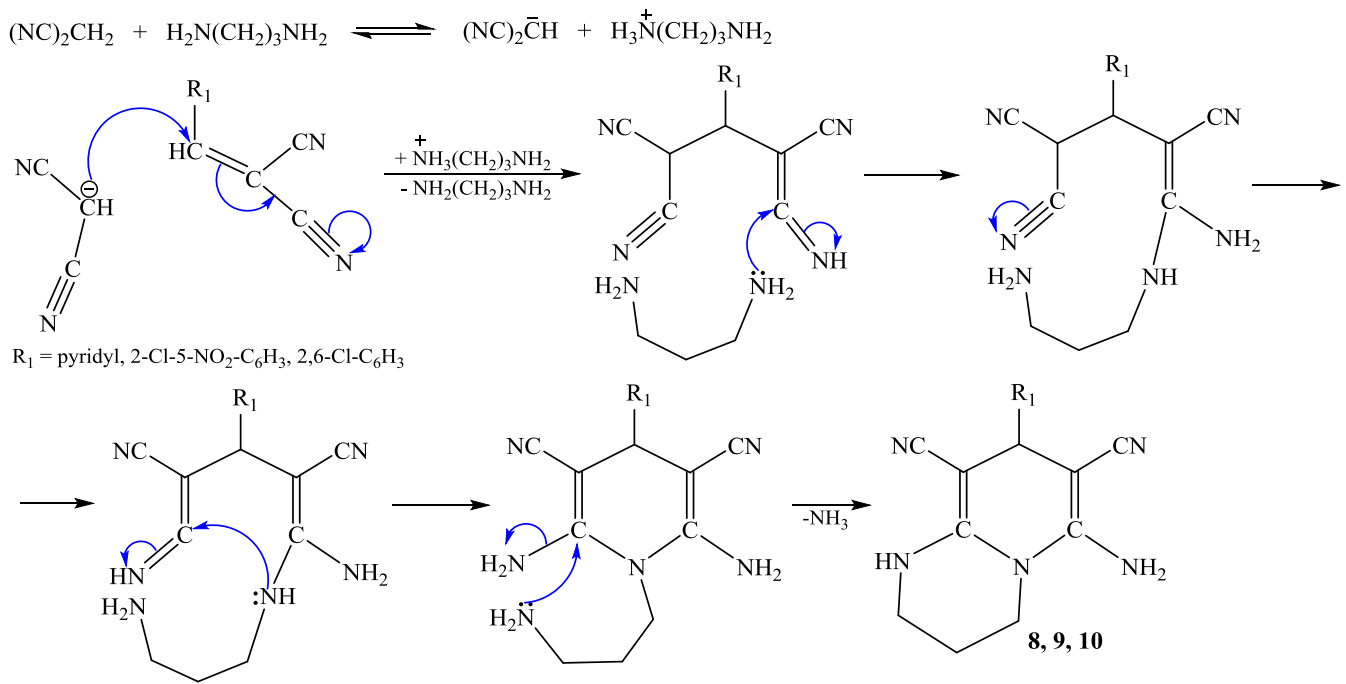


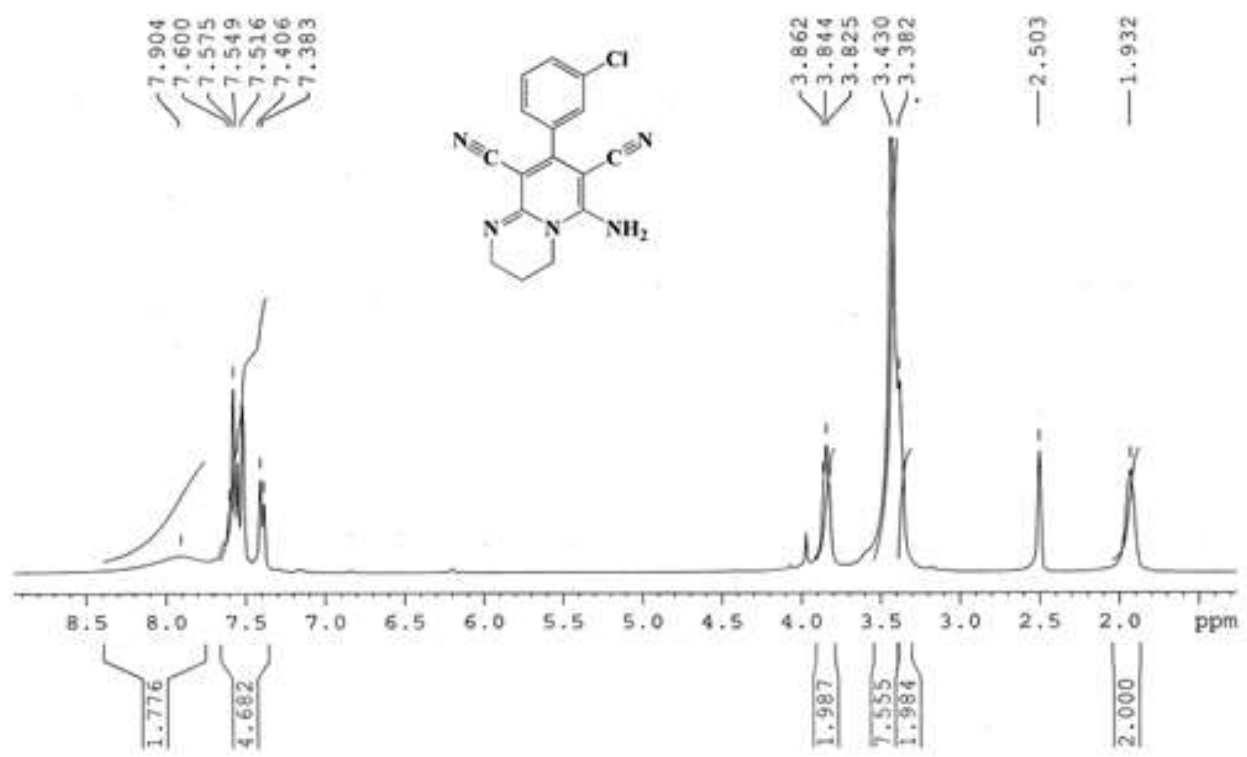

Fig. 1. ${ }^{1} \mathrm{H}$ NMR spectrum of 6-amino-8-(3-chlorophenyl)-3,4-dihydro-2H-pyrido[1,2-a]pyrimidine-7,9-dicarbonitrile (4e).

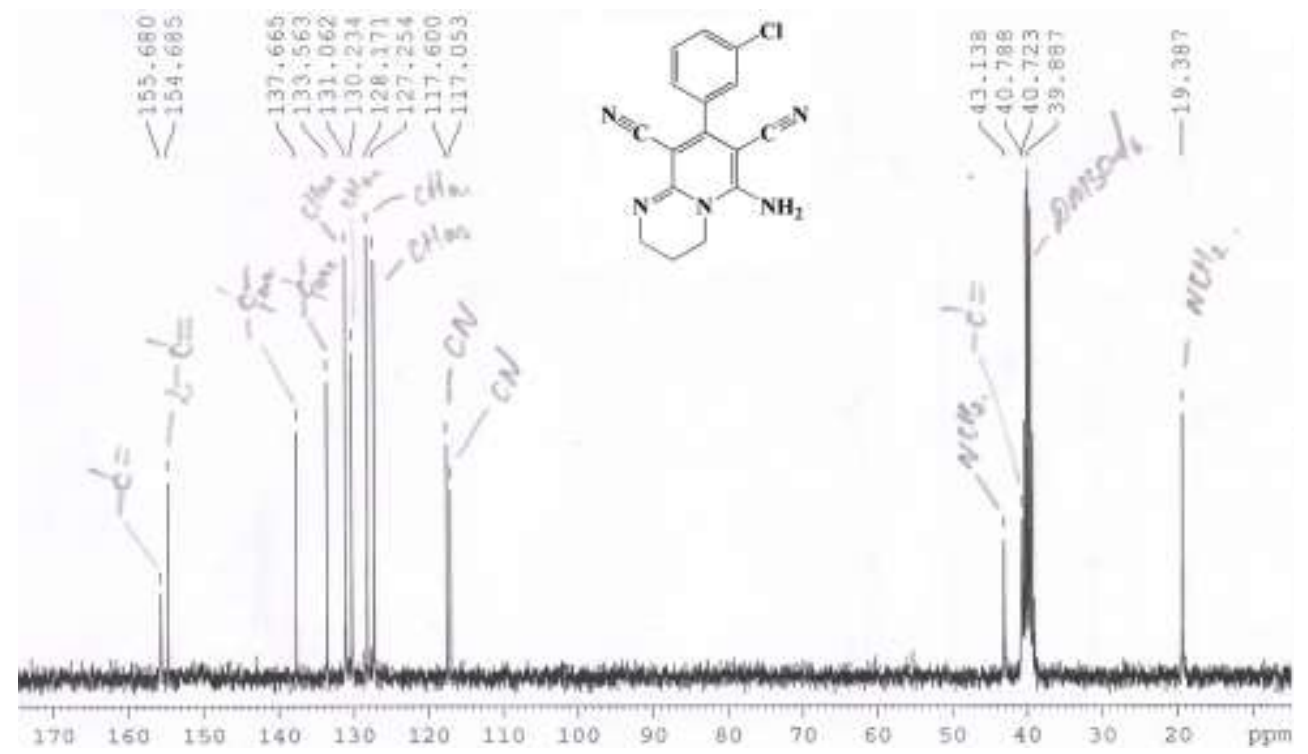

Fig. 2. ${ }^{13} \mathrm{C}$ NMR spectrum of 6-amino-8-(3-chlorophenyl)-3,4-dihydro-2H-pyrido[1,2-a]pyrimidine-7,9-dicarbonitrile (4e).

\section{Experimental part. General remarks}

All commercially available chemicals were obtained from Merck and Fluka (Sigma Aldrich) companies and used without further purification. Melting points were measured on Stuart SMP30 apparatus without correction. ${ }^{1} \mathrm{H},{ }^{13} \mathrm{C}$ NMR spectra were recorded on Bruker Avance $300-\mathrm{MHz}$ spectrometer at 300 and 75 $\mathrm{MHz}$, respectively. Thin-layer chromatography (TLC) on commercial aluminum-backed plates of silica gel (60 F254) was used to mo nitor the course of reactions.

\section{General experimental procedure}

6-Amino-8-phenyl-3,4-dihydro-2Hpyrido[1,2-a]pyrimidine-7,9-dicarbonitrile (4a): Benzylidenecyanoacetamide, $(4 \mathrm{mmol})$ malononitril (4.1 $\mathrm{mmol})$ and 1,3diaminopropane $(4.1 \mathrm{mmol})$ stirrered in $35 \mathrm{ml}$ of methyl alcohol. Then the reaction mixture is maintained at room temperature for 2 days. The course of the reaction was monitored by 
TLC (EtOAc/n-hexane, 3:2). Crystals were precipitated after evaporation of solvent, filtered by paper, recrystallized from ethanol-water mixture and obtained in pure form (yield 0.81 $\mathrm{g}, 72.97 \%)$. $\mathrm{T}_{\mathrm{mp}}=234^{\circ} \mathrm{C}$.

${ }^{1} \mathrm{H}$ NMR (300 MHz, DMSO-d6): 1.94 (t, $\left.2 \mathrm{H}, \mathrm{CH}_{2},{ }^{3} J_{\mathrm{H}-\mathrm{H}}=6,6\right) ; 3.38\left(\mathrm{t}, 2 \mathrm{H}, \mathrm{CH}_{2},{ }^{3} J_{\mathrm{H}-}\right.$ $\mathrm{H}=6,3) ; 3.86\left(\mathrm{t}, 2 \mathrm{H}, \mathrm{CH}_{2},{ }^{3} J_{\mathrm{H}-\mathrm{H}}=5,7\right) ; 7.41-$ $7.52(\mathrm{~m}, 5 \mathrm{H}, 5 \mathrm{Ar}-\mathrm{H}) ; 7.84\left(\mathrm{~s}, 2 \mathrm{H}, \mathrm{NH}_{2}\right) .{ }^{13} \mathrm{C}$ NMR spektr (DMSO-d6), $\delta, \quad$ m.h.: 19.39 $\left(\mathrm{CH}_{2}\right), 40.72\left(2=\mathrm{C}_{\text {quat. }}\right), 42.99\left(\mathrm{CH}_{2}\right), 43.04$ $\left(\mathrm{CH}_{2}\right), \quad 117.25 \quad(\mathrm{CN}), \quad 117.85 \quad(\mathrm{CN}), 128.39$ $\left(2 \mathrm{CH}_{\text {arom }}\right), 128.99\left(2 \mathrm{CH}_{\text {arom }}\right), 130.30\left(\mathrm{CH}_{\text {arom }}\right)$, $135.67\left(\mathrm{C}_{\mathrm{ar}}\right), 155.81$ ( $\left.=\mathrm{C}_{\text {quat. }}\right), 156.27\left(2=\mathrm{C}_{\text {quat. }}\right)$.

Found, \%: $69.76 \mathrm{C} ; 4.68 \mathrm{H}, 25.50 \mathrm{~N}$. $\mathrm{C}_{16} \mathrm{H}_{13} \mathrm{~N}_{5}$. Calculated, \%: $69.82 \mathrm{C} ; 4.73 \mathrm{H}, 25.45$ $\mathrm{N}$.

6-Amino-8-(p-tolyl)-3,4-dihydro-2Hpyrido[1,2-a]pyrimidine-7,9-dicarbonitrile

(4b): Synthesized by the same way(yield 0.88 $\mathrm{g}, 75.86 \%) . \mathrm{T}_{\mathrm{mp} .}=250^{\circ} \mathrm{C}$.

${ }^{1} \mathrm{H}$ NMR (300 MHz, DMSO-d6): 1.93 (t, $\left.2 \mathrm{H}, \mathrm{CH}_{2},{ }^{3} J_{\mathrm{H}-\mathrm{H}}=6,7\right) ; 2.37$ (s, 3H, $\left.\mathrm{CH}_{3}-\mathrm{Ar}\right)$; $3.37\left(\mathrm{t}, 2 \mathrm{H}, \mathrm{CH}_{2},{ }^{3} J_{\mathrm{H}-\mathrm{H}}=6,3\right) ; 3.85(\mathrm{t}, 2 \mathrm{H}$, $\left.\mathrm{CH}_{2},{ }^{3} J_{\mathrm{H}-\mathrm{H}}=6,9\right) ; 7.31(\mathrm{~m}, 4 \mathrm{H}, 4 \mathrm{Ar}-\mathrm{H}) ; 7.82$ $\left(\mathrm{s}, 2 \mathrm{H}, \mathrm{NH}_{2}\right) .{ }^{13} \mathrm{C}$ NMR spektr (DMSO-d6), $\delta$, m.h.: $19.37\left(\mathrm{CH}_{2}\right), 21.37\left(\mathrm{Ar}-\mathrm{CH}_{3}\right), 40.54$ $\left(\mathrm{CH}_{2}\right), 40.66$ (2=C $\left.\mathrm{C}_{\text {quat. }}\right), 42.99\left(\mathrm{CH}_{2}\right), 117.33$ $(\mathrm{CN}), 117.96(\mathrm{CN}), 128.35\left(2 \mathrm{CH}_{\text {arom }}\right), 129.52$ $\left(2 \mathrm{CH}_{\text {arom }}\right), 132.72\left(\mathrm{C}_{\mathrm{ar}}\right), 140.08\left(\mathrm{C}_{\mathrm{ar}}\right), 155.85$ $\left(=\mathrm{C}_{\text {quat. }}\right), 156.31\left(2=\mathrm{C}_{\text {quat. }}\right)$.

Found, \%: $70.64 \mathrm{C} ; 5.25 \mathrm{H}, 24.16 \mathrm{~N}$. $\mathrm{C}_{17} \mathrm{H}_{15} \mathrm{~N}_{5}$. Calculated, \%: $70.59 \mathrm{C} ; 5.19 \mathrm{H}, 24.22$ $\mathrm{N}$.

6-Amino-8-(4-methoxyphenyl)-3,4dihydro-2H-pyrido[1,2-a]pyrimidine-7,9-dicarbonitrile (4c): Synthesized by the same way (yield $0.9 \mathrm{~g}, 73.17 \%$ ). $\mathrm{T}_{\mathrm{mp} .}=231^{\circ} \mathrm{C}$.

${ }^{1} \mathrm{H}$ NMR (300 MHz, DMSO-d6): 1.93 (s, 2H, $\left.\mathrm{CH}_{2}\right) ; 3.37\left(\mathrm{~s}, 2 \mathrm{H}, \mathrm{CH}_{2}\right) ; 3.81(\mathrm{~s}, 3 \mathrm{H}$, $\left.\mathrm{CH}_{3} \underline{\mathrm{O}}-\mathrm{Ar}\right) ; 3.85\left(\mathrm{~s}, 2 \mathrm{H}, \mathrm{CH}_{2}\right) ; 7.05(\mathrm{~d}, 2 \mathrm{H}$, $\left.2 \mathrm{Ar}-\mathrm{H},{ }^{3} J_{\mathrm{H}-\mathrm{H}}=8,1\right) ; 7.39\left(\mathrm{~d}, 2 \mathrm{H}, 2 \mathrm{Ar}-\mathrm{H},{ }^{3} J_{\mathrm{H}-\mathrm{H}}\right.$ $=7,8) ; 7.80\left(\mathrm{~s}, 2 \mathrm{H}, \mathrm{NH}_{2}\right) .{ }^{13} \mathrm{C} \mathrm{NMR}$ spektr (DMSO-d6), $\delta$, m.h.: $19.40 \quad\left(\mathrm{CH}_{2}\right), 38.91$ $\left(2=\mathrm{C}_{\text {quat. }}\right), 40.52\left(\mathrm{CH}_{2}\right), 42.99\left(\mathrm{CH}_{2}\right), 55.73$ $\left(\mathrm{CH}_{3} \mathrm{O}\right), \quad 114.28 \quad\left(2 \mathrm{CH}_{\text {arom }}\right), \quad 117.50 \quad(\mathrm{CN})$, $118.13(\mathrm{CN}), 127.58\left(\mathrm{C}_{\mathrm{ar}}\right), 127.59\left(=\mathrm{C}_{\text {quat. }}\right)$, $130.12\left(2 \mathrm{CH}_{\text {arom }}\right), 155.88 \quad\left(=\mathrm{C}_{\text {quat. }}\right), 155.94$ $\left(=\mathrm{C}_{\text {quat. }}\right), 160.85\left(\mathrm{O}-\underline{\mathrm{C}}_{\mathrm{ar}}\right)$.
Found, \%: $66.82 \mathrm{C} ; 4.87 \mathrm{H}, 23.01 \mathrm{~N}$. $\mathrm{C}_{17} \mathrm{H}_{15} \mathrm{~N}_{5} \mathrm{O}$. Calculated, \%: $66.88 \mathrm{C} ; 4.92 \mathrm{H}$, $22.95 \mathrm{~N}$.

6-Amino-8-(4-fluorophenyl)-3,4dihydro-2H-pyrido[1,2-a]pyrimidine-7,9dicarbonitrile (4d): Synthesized by the same way (yield $0.79 \mathrm{~g}, 67.52 \%$ ). $\mathrm{T}_{\mathrm{mp}} .=238^{\circ} \mathrm{C}$.

${ }^{1} \mathrm{H}$ NMR (300 MHz, DMSO-d6): 1.93 $\left(\mathrm{s}, 2 \mathrm{H}, \mathrm{CH}_{2}\right) ; 3.38\left(\mathrm{~s}, 2 \mathrm{H}, \mathrm{CH}_{2}\right) ; 3.85(\mathrm{~s}, 2 \mathrm{H}$, $\left.\mathrm{CH}_{2}\right) ; 7.12-7.52(\mathrm{~m}, 4 \mathrm{H}, 4 \mathrm{Ar}-\mathrm{H}) ; 7.96(\mathrm{~s}, 2 \mathrm{H}$, $\mathrm{NH}_{2}$ ). ${ }^{13} \mathrm{C}$ NMR spektr (DMSO-d6), $\delta$, m.h.: $19.37\left(\mathrm{CH}_{2}\right), 39.02$ (2=C $\left.\mathrm{C}_{\text {quat. }}\right), 40.68\left(\mathrm{CH}_{2}\right)$, $43.06\left(\mathrm{CH}_{2}\right), 115.91-116.20\left(2 \mathrm{CH}_{\text {arom }}\right), 117.22$ $(\mathrm{CN}), 117.78(\mathrm{CN}), 130.89-131.00\left(2 \mathrm{CH}_{\text {arom }}\right)$, $132.02 \quad\left(\mathrm{C}_{\mathrm{ar}}\right), \quad 155.33 \quad\left(2=\mathrm{C}_{\text {quat. }}\right), \quad 155.75$ $\left(=\mathrm{C}_{\text {quat. }}\right), 161.66-164.92\left(\mathrm{~F}-\underline{\mathrm{C}}_{\mathrm{ar}}\right)$.

Found, \%: $65.59 \mathrm{C} ; 4.14 \mathrm{H}, 23.84 \mathrm{~N}$. $\mathrm{C}_{16} \mathrm{H}_{12} \mathrm{~N}_{5} \mathrm{~F}$. Calculated, \%: $65.53 \mathrm{C} ; 4.09 \mathrm{H}$, $23.89 \mathrm{~N}$.

6-Amino-8-(3-chlorophenyl)-3,4dihydro-2H-pyrido[1,2-a]pyrimidine-7,9-

dicarbonitrile (4e): Synthesized by the same way (yield $0.91 \mathrm{~g}, 73.39 \%$ ). $\mathrm{T}_{\mathrm{mp} .}=254^{\circ} \mathrm{C}$.

${ }^{1} \mathrm{H}$ NMR (300 MHz, DMSO-d6): 1.93 $\left(\mathrm{s}, 2 \mathrm{H}, \mathrm{CH}_{2}\right) ; 3.38\left(\mathrm{~s}, 2 \mathrm{H}, \mathrm{CH}_{2}\right) ; 3.84(\mathrm{~s}, 2 \mathrm{H}$, $\left.\mathrm{CH}_{2}\right)$; 7.38-7.60 (m, 4H, 4Ar-H); 7.90 (s, 2H, $\mathrm{NH}_{2}$ ). ${ }^{13} \mathrm{C}$ NMR spektr (DMSO-d6), $\delta$, m.h.: $19.39\left(\mathrm{CH}_{2}\right), 40.72\left(\mathrm{CH}_{2}\right), 40.79\left(2=\mathrm{C}_{\text {quat. }}\right)$, $43.14\left(\mathrm{CH}_{2}\right), \quad 117.05(\mathrm{CN}), 117.60(\mathrm{CN})$, $127.25\left(\mathrm{CH}_{\text {arom }}\right), \quad 128.17 \quad\left(\mathrm{CH}_{\text {arom }}\right), \quad 130.23$ $\left(\mathrm{CH}_{\text {arom }}\right), \quad 131.06 \quad\left(\mathrm{CH}_{\text {arom }}\right), 133.56 \quad\left(\mathrm{C}_{\mathrm{ar}}\right)$, $137.66 \quad\left(\mathrm{C}_{\mathrm{ar}}\right), \quad 154.68 \quad\left(2=\mathrm{C}_{\text {quat. }}\right), \quad 155.68$ $\left(=\mathrm{C}_{\text {quat. }}\right)$.

Found, \%: $62.09 \mathrm{C} ; 3.93 \mathrm{H}, 22.56 \mathrm{~N}$. $\mathrm{C}_{16} \mathrm{H}_{12} \mathrm{~N}_{5} \mathrm{Cl}$. Calculated, \%: $62.03 \mathrm{C} ; 3.88 \mathrm{H}$, $22.62 \mathrm{~N}$.

6-Amino-8-(4-bromophenyl)-3,4dihydro-2H-pyrido[1,2-a]pyrimidine-7,9-dicarbonitrile (4f): Synthesized by the same way (yield $0.89 \mathrm{~g}, 62.68 \%$ ). $\mathrm{T}_{\mathrm{mp} .}=278^{\circ} \mathrm{C}$.

${ }^{1} \mathrm{H}$ NMR (300 MHz, DMSO-d6): 1.93 (s, 2H, $\left.\mathrm{CH}_{2}\right) ; 3.37\left(\mathrm{~s}, 2 \mathrm{H}, \mathrm{CH}_{2}\right) ; 3.83(\mathrm{~s}, 2 \mathrm{H}$, $\left.\mathrm{CH}_{2}\right) ; 7.38\left(\mathrm{~d}, 2 \mathrm{H}, 2 \mathrm{Ar}-\mathrm{H},{ }^{3} J_{\mathrm{H}-\mathrm{H}}=8,1\right) ; 7.73(\mathrm{~d}$, $\left.2 \mathrm{H}, 2 \mathrm{Ar}-\mathrm{H},{ }^{3} \mathrm{~J}_{\mathrm{H}-\mathrm{H}}=8,1\right) ; 7.93\left(\mathrm{~s}, 2 \mathrm{H}, \mathrm{NH}_{2}\right) .{ }^{13} \mathrm{C}$ NMR spektr (DMSO-d6), $\delta, \quad$ m.h.: 19.37 $\left(\mathrm{CH}_{2}\right), 40.69\left(\mathrm{CH}_{2}\right), 40.72 \quad\left(2=\mathrm{C}_{\text {quat. }}\right), 43.15$ $\left(\mathrm{CH}_{2}\right), 117.15(\mathrm{CN}), 117.69(\mathrm{CN}), 123.85(\mathrm{Br}-$ $\left.\underline{\mathrm{C}}_{\mathrm{ar}}\right), 130.60 \quad\left(2 \mathrm{CH}_{\text {arom }}\right), 132.09 \quad\left(2 \mathrm{CH}_{\text {arom }}\right)$, $134.87 \quad\left(\mathrm{C}_{\mathrm{ar}}\right), \quad 155.15 \quad\left(2=\mathrm{C}_{\text {quat. }}\right), \quad 155.67$ $\left(=\mathrm{C}_{\text {quat. }}\right)$. 
Found, \%: $54.19 \mathrm{C} ; 3.33 \mathrm{H}, 19.82 \mathrm{~N}$. $\mathrm{C}_{16} \mathrm{H}_{12} \mathrm{~N}_{5} \mathrm{Br}$. Calculated, \%: $54.24 \mathrm{C} ; 3.39 \mathrm{H}$, $19.77 \mathrm{~N}$.

\section{6-Amino-8-(4-}

(trifluoromethyl)phenyl)-3,4-dihydro-2Hpyrido[1,2-a]pyrimidine-7,9-dicarbonitrile (4g): Synthesized by the same way (yield 0.9 $\mathrm{g}, 65.69 \%) . \mathrm{T}_{\mathrm{mp}}=331{ }^{\circ} \mathrm{C}$.

${ }^{1} \mathrm{H}$ NMR (300 MHz, DMSO-d6): 1.94 (s, 2H, $\left.\mathrm{CH}_{2}\right) ; 3.38\left(\mathrm{~s}, 2 \mathrm{H}, \mathrm{CH}_{2}\right) ; 3.84(\mathrm{~s}, 2 \mathrm{H}$, $\left.\mathrm{CH}_{2}\right) ; 7.66\left(\mathrm{~d}, 2 \mathrm{H}, 2 \mathrm{Ar}-\mathrm{H},{ }^{3} \mathrm{~J}_{\mathrm{H}-\mathrm{H}}=7,8\right) ; 7.90(\mathrm{~d}$, $\left.2 \mathrm{H}, 2 \mathrm{Ar}-\mathrm{H},{ }^{3} \mathrm{~J}_{\mathrm{H}-\mathrm{H}}=7,8\right) ; 7.96\left(\mathrm{~s}, 2 \mathrm{H}, \mathrm{NH}_{2}\right) .{ }^{13} \mathrm{C}$ NMR spektr (DMSO-d6), $\delta$, m.h.: 19.35 $\left(\mathrm{CH}_{2}\right), 40.62\left(\mathrm{CH}_{2}\right), 40.75\left(2=\mathrm{C}_{\text {quat. }}\right), 43.19$ $\left(\mathrm{CH}_{2}\right), 117.02(\mathrm{CN}), 117.54(\mathrm{CN}), 126.03-$ $126.17\left(2 \mathrm{CH}_{\text {arom }}\right), 129.50\left(2 \mathrm{CH}_{\text {arom }}\right), 130.28$ $\left(\mathrm{CF}_{3}-\underline{\mathrm{C}}_{\mathrm{ar}}\right), 130.70\left(\mathrm{CF}_{3}\right), 139.80\left(\mathrm{C}_{\mathrm{ar}}\right), 154.93$ $\left(2=\mathrm{C}_{\text {quat. }}\right), 155.66\left(=\mathrm{C}_{\text {quat. }}\right)$.

Found, \%: $59.41 \mathrm{C} ; 3.45 \mathrm{H}, 20.47 \mathrm{~N}$. $\mathrm{C}_{17} \mathrm{H}_{12} \mathrm{~N}_{5} \mathrm{~F}_{3}$. Calculated, \%: $59.47 \mathrm{C} ; 3.50 \mathrm{H}$, $20.41 \mathrm{~N}$.

6-Amino-8-(4-hydroxy-3-

methoxyphenyl)-3,4-dihydro-2H-pyrido[1,2a]pyrimidine-7,9-dicarbonitrile (4h): Synthesized by the same way (yield $0.93 \mathrm{~g}, 72.66 \%$ ). $\mathrm{T}_{\mathrm{mp} .}=181^{\circ} \mathrm{C}$.

${ }^{1} \mathrm{H}$ NMR (300 MHz, DMSO-d6): 1.93 (s, 2H, $\left.\mathrm{CH}_{2}\right) ; 3.36\left(\mathrm{~s}, 2 \mathrm{H}, \mathrm{CH}_{2}\right) ; 3.78(\mathrm{~s}, 3 \mathrm{H}$, $\left.\mathrm{CH}_{3} \mathrm{O}\right) ; 3.85\left(\mathrm{~s}, 2 \mathrm{H}, \mathrm{CH}_{2}\right) ; 6.86-7.61(\mathrm{~m}, 6 \mathrm{H}$, $\left.3 \mathrm{Ar}-\mathrm{H}+\mathrm{Ar}-\mathrm{OH}+\mathrm{NH}_{2}\right) . \quad{ }^{13} \mathrm{C} \quad \mathrm{NMR}$ spektr (DMSO-d6), $\delta$, m.h.: $19.53\left(\mathrm{CH}_{2}\right), 40.62\left(\mathrm{CH}_{2}\right)$, $42.85\left(\mathrm{CH}_{2}\right), 56.13\left(\underline{\mathrm{CH}_{3}} \mathrm{O}\right), 76.70\left(=\mathrm{C}_{\text {quat. }}\right)$, $80.42 \quad\left(=\mathrm{C}_{\text {quat }}\right), \quad 112.88 \quad\left(\mathrm{CH}_{\text {arom }}\right), \quad 115.80$ $\left(\mathrm{CH}_{\text {arom }}\right), 117.80(\mathrm{CN}), 118.52(\mathrm{CN}), 121.81$ $\left(\mathrm{CH}_{\text {arom }}\right), 125.67\left(\mathrm{C}_{\mathrm{ar}}\right), 147.70$ (= $\left.\mathrm{C}_{\text {quat. }}\right), 149.42$ $\left(=\mathrm{C}_{\text {quat. }}\right), \quad 151.57 \quad\left(=\mathrm{C}_{\text {quat. }}\right), \quad 156.14 \quad\left(\mathrm{O}-\mathrm{C}_{\mathrm{ar}}\right)$, $156.24\left(\mathrm{O}-\mathrm{C}_{\mathrm{ar}}\right)$.

Found, \%: $63.60 \mathrm{C} ; 4.73 \mathrm{H}, 21.76 \mathrm{~N}$. $\mathrm{C}_{17} \mathrm{H}_{15} \mathrm{~N}_{5} \mathrm{O}_{2}$. Calculated, \%: $63.55 \mathrm{C} ; 4.67 \mathrm{H}$, $21.81 \mathrm{~N}$.

6-Amino-8-(thiophen-2-yl)-3,4dihydro-2H-pyrido[1,2-a]pyrimidine-7,9dicarbonitrile (4j): Synthesized by the same way (yield $0.76 \mathrm{~g}, 67.86 \%$ ). $\mathrm{T}_{\mathrm{mp}} .=227^{\circ} \mathrm{C}$.

${ }^{1} \mathrm{H}$ NMR (300 MHz, DMSO-d6): 1.93 (m, 2H, $\left.\mathrm{CH}_{2}\right) ; 3.37$ (s, 2H, $\left.\mathrm{CH}_{2}\right) ; 3.84(\mathrm{~s}, 2 \mathrm{H}$, $\left.\mathrm{CH}_{2}\right) ; 7.21\left(\mathrm{t}, 1 \mathrm{H}\right.$, Thioph-H, $\left.{ }^{3} J_{\mathrm{H}-\mathrm{H}}=4,2\right) ; 7.41$ $\left(\mathrm{d}, 1 \mathrm{H}\right.$, Thioph-H, $\left.{ }^{3} J_{\mathrm{H}-\mathrm{H}}=3\right) ; 7.76(\mathrm{~s}, 2 \mathrm{H}$, $\left.\mathrm{NH}_{2}\right) ; 7.83\left(\mathrm{~d}, 1 \mathrm{H}\right.$, Thioph-H, $\left.{ }^{3} J_{\mathrm{H}-\mathrm{H}}=4.8\right) .{ }^{13} \mathrm{C}$ NMR spektr (DMSO-d6), $\delta$, m.h.: 19.34 $\left(\mathrm{CH}_{2}\right), 40.68$ (2=C $\left.\mathrm{C}_{\text {quat. }}\right), 40.72 \quad\left(\mathrm{CH}_{2}\right), 43.18$
$\left(\mathrm{CH}_{2}\right), 117.27 \quad(\mathrm{CN}), \quad 117.89 \quad(\mathrm{CN}), 127.93$

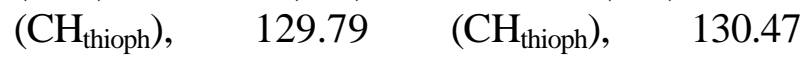
$\left(\mathrm{CH}_{\text {thioph }}\right), 134.67 \quad\left(\mathrm{C}_{\text {thioph }}\right), \quad 148.41 \quad\left(=\mathrm{C}_{\text {quat. }}\right)$, 155.70 ( $=\mathrm{C}_{\text {quat }}$ ), 162.35 ( $=\mathrm{C}_{\text {quat. }}$ ).

Found, \%: $59.74 \mathrm{C} ; 3.85 \mathrm{H}, 24.96 \mathrm{~N}$. $\mathrm{C}_{14} \mathrm{H}_{11} \mathrm{~N}_{5} \mathrm{~S}$. Calculated, \%: $59.79 \mathrm{C} ; 3.91 \mathrm{H}$, 24.91N.

6-Amino-8-(pyridin-4-yl)-1,3,4,8-

tetrahydro-2H-pyrido[1,2-a]pyrimidine-7,9dicarbonitrile (6): Synthesized by the same way (yield $0.85 \mathrm{~g}, 76.57 \%$ ). $\mathrm{T}_{\mathrm{mp} .}=242^{\circ} \mathrm{C}$.

${ }^{1} \mathrm{H}$ NMR (300 MHz, DMSO-d6): 1.88 $\left(\mathrm{m}, 2 \mathrm{H}, \mathrm{CH}_{2}\right) ; 3.13\left(\mathrm{~m}, 2 \mathrm{H}, \mathrm{CH}_{2}\right) ; 3.60(\mathrm{~m}$, $\left.2 \mathrm{H}, \mathrm{CH}_{2}\right) ; 4.06(\mathrm{~s}, 1 \mathrm{H}, \underline{\mathrm{CH}}-\mathrm{Ar}) ; 6.27(\mathrm{~s}, 2 \mathrm{H}$, $\left.\mathrm{NH}_{2}\right) ; 6.90$ (s, 1H, NH); 7.18 (m, 2H, 2Ar-H); $8.51(\mathrm{~m}, 2 \mathrm{H}, 2 \mathrm{Ar}-\mathrm{H}) .{ }^{13} \mathrm{C}$ NMR spektr (DMSOd6), $\delta$, m.h.: $22.01\left(\mathrm{CH}_{2}\right), 38.61\left(\mathrm{CH}_{2}\right), 38.94$ (Ar- $-\mathrm{CH}), 43.07\left(\mathrm{CH}_{2}\right), 54.05\left(=\mathrm{C}_{\text {quat. }}\right), 57.64$ $\left(=\mathrm{C}_{\text {quat. }}\right), 121.90 \quad(2 \mathrm{CN}), 122.28 \quad\left(2 \mathrm{CH}_{\text {arom }}\right)$, $150.31 \quad\left(2 \mathrm{CH}_{\text {arom }}\right), \quad 151.26 \quad\left(\mathrm{C}_{\mathrm{ar}}\right), \quad 153.21$ $\left(=\mathrm{C}_{\text {quat. }}\right), 155.15\left(=\mathrm{C}_{\text {quat. }}\right)$.

Found, \%: $64.69 \mathrm{C} ; 4.98 \mathrm{H}, 30.26 \mathrm{~N}$. $\mathrm{C}_{15} \mathrm{H}_{14} \mathrm{~N}_{6}$. Calculated, \%: 64.75 C; $5.03 \mathrm{H}, 30.21$ $\mathrm{N}$.

6-Amino-8-(2-chloro-5-nitrophenyl)1,3,4,8-tetrahydro-2H-pyrido[1,2-a]pyrimidine-7,9-dicarbonitrile (8): Synthesized by the same way (yield $1 \mathrm{~g}, 69.93 \%$ ). $\mathrm{T}_{\mathrm{mp}}$. = $243^{\circ} \mathrm{C}$.

${ }^{1} \mathrm{H}$ NMR (300 MHz, DMSO-d6): 1.90 (m, 2H, $\left.\mathrm{CH}_{2}\right) ; 3.18\left(\mathrm{~m}, 2 \mathrm{H}, \mathrm{CH}_{2}\right) ; 3.64(\mathrm{~s}, 2 \mathrm{H}$, $\left.\mathrm{CH}_{2}\right) ; 4.73$ (s, 1H, $\left.\underline{\mathrm{CH}}-\mathrm{Ar}\right) ; 6.36\left(\mathrm{~s}, 2 \mathrm{H}, \mathrm{NH}_{2}\right)$; $7.00(\mathrm{~s}, 1 \mathrm{H}, \mathrm{NH}) ; 7.71(\mathrm{~d}, 1 \mathrm{H}, \mathrm{Ar}-\mathrm{H}) ; 8.07$ (d, 2H, 2Ar-H). ${ }^{13} \mathrm{C}$ NMR spektr (DMSO-d6), $\delta$, m.h.: $22.12\left(\mathrm{CH}_{2}\right), 37.14(\mathrm{Ar}-\mathrm{CH}), 38.64$ $\left(\mathrm{CH}_{2}\right), \quad 43.20 \quad\left(\mathrm{CH}_{2}\right), \quad 53.64 \quad\left(=\mathrm{C}_{\text {quat. }}\right), \quad 57.02$ $\left(=\mathrm{C}_{\text {quat. }}\right), 121.48(\mathrm{CN}), 121.89(\mathrm{CN}), 123.65$ $\left(\mathrm{CH}_{\text {arom }}\right), 124.58\left(\mathrm{CH}_{\text {arom }}\right), 131.64\left(\mathrm{CH}_{\text {arom }}\right)$, $138.68\left(\mathrm{C}_{\mathrm{ar}}\right), 145.56\left(\mathrm{C}_{\mathrm{ar}}\right), 147.37\left(\mathrm{C}_{\mathrm{ar}}\right), 151.69$ $\left(=\mathrm{C}_{\text {quat. }}\right), 153.70\left(=\mathrm{C}_{\text {quat. }}\right)$.

Found, \%: $53.91 \mathrm{C} ; 3.71 \mathrm{H}, 23.50 \mathrm{~N}$. $\mathrm{C}_{16} \mathrm{H}_{13} \mathrm{~N}_{6} \mathrm{O}_{2} \mathrm{Cl}$. Calculated, \%: $53.86 \mathrm{C} ; 3.65 \mathrm{H}$, $23.56 \mathrm{~N}$.

6-Amino-8-(2,6-dichlorophenyl)-

1,3,4,8-tetrahydro-2H-pyrido[1,2-

a]pyrimidine-7,9-dicarbonitrile (10): Synthesized by the same way (yield $1.07 \mathrm{~g}$, $77.54 \%) . \mathrm{T}_{\mathrm{mp} .}=268^{\circ} \mathrm{C}$.

${ }^{1} \mathrm{H}$ NMR (300 MHz, DMSO-d6): 1.89 $\left(\mathrm{m}, 2 \mathrm{H}, \mathrm{CH}_{2}\right) ; 3.13\left(\mathrm{~m}, 2 \mathrm{H}, \mathrm{CH}_{2}\right) ; 3.67(\mathrm{~s}, 2 \mathrm{H}$, 
$\left.\mathrm{CH}_{2}\right) ; 5.31$ (s, 1H, $\left.\underline{\mathrm{CH}}-\mathrm{Ar}\right) ; 6.14\left(\mathrm{~s}, 2 \mathrm{H}, \mathrm{NH}_{2}\right)$; $6.78(\mathrm{~s}, 1 \mathrm{H}, \mathrm{NH}) ; 7.25\left(\mathrm{t}, 1 \mathrm{H}, \mathrm{Ar}-\mathrm{H},{ }^{3} J_{\mathrm{H}-\mathrm{H}}=\right.$ $8,1) ; 7.42\left(\mathrm{~d}, 2 \mathrm{H}, 2 \mathrm{Ar}-\mathrm{H},{ }^{3} \mathrm{~J}_{\mathrm{H}-\mathrm{H}}=7,8\right) .{ }^{13} \mathrm{C}$ NMR spektr (DMSO-d6), $\delta$, m.h.: 22.30 $\left(\mathrm{CH}_{2}\right), 36.32(\mathrm{Ar}-\underline{\mathrm{CH}}), 38.62\left(\mathrm{CH}_{2}\right), 42.92$ $\left(\mathrm{CH}_{2}\right), 51.70$ (= $\left.\mathrm{C}_{\text {quat. }}\right), 55.06$ (= $\left.\mathrm{C}_{\text {quat. }}\right), 121.61$
$(\mathrm{CN}), 122.04(\mathrm{CN}), 129.56\left(3 \mathrm{CH}_{\text {arom }}\right), 138.25$

$\left(\mathrm{C}_{\mathrm{ar}}\right), 152.11\left(\mathrm{Cl}-\underline{\mathrm{C}}_{\mathrm{ar}}\right), 152.12$ (=C $\left.\mathrm{C}_{\text {quat. }}\right), 154.16$ $\left(=\mathrm{C}_{\text {quat. }}\right), 154.17\left(\mathrm{Cl}-\underline{\mathrm{C}}_{\mathrm{ar}}\right)$.

Found, \%: $55.43 \mathrm{C} ; 3.71 \mathrm{H}, 20.28 \mathrm{~N}$. $\mathrm{C}_{16} \mathrm{H}_{13} \mathrm{~N}_{5} \mathrm{Cl}_{2}$. Calculated, \%: $55.49 \mathrm{C} ; 3.76 \mathrm{H}$, $20.23 \mathrm{~N}$.

This study was performed under financial support by the Baku State University (grant 50+50).

\section{References}

1. Dilip D. Dhavale, Mohammed M. Matin, Tarun Sharma and Sushma G. Sabharwal. Synthesis and evaluation of glycosidase inhibitory activity of octahydro- $2 \mathrm{H}$ pyrido[1,2-a]pyrimidine and octahydroimidazo[1,2-a]pyridine bicyclic diazasugars. Bioorganic \& Medicinal Chemistry. 2004, vol.12, iss.15, pp. 4039-4044. DOI: 10.1016/j.bmc.2004.05.030.

2. Monica Donghi, Olaf D. Kinzel, Vincenzo Summa. 3-Hydroxy-4-oxo-4H-pyrido[1,2a]pyrimidine-2-carboxylates-A new class of HIV-1 integrase inhibitors. Bioorganic \& Medicinal Chemistry Letters. 2009, vol.19, iss.7, pp. 1930-1934. DOI: 10.1016/j.bmcl.2009.02.055.

3. Olaf D.Kinzel, Richard G.Ball, Monica Donghi, Courtney K.Maguire, Ester Muraglia, Silvia Pesci, Michael Rowley, Vincenzo Summa. 3-Hydroxy-4-oxo-4Hpyrido[1,2-a]pyrimidine-2-carboxylatesfast access to a heterocyclic scaffold for HIV-1 integrase inhibitors. Tetrahedron Letters. 2008, vol.49, iss.46, p.6556-6558. DOI:10.1016/j.tetlet.2008.09.010.

4. Ping Gao, Lingzi Zhang, Lin Sun, Tianguang Huang, Jing Tan, Jian Zhang, Zhongxia Zhou, Tong Zhao, Luis Menéndez-Arias, Christophe Pannecouque, Erik De Clercq, Peng Zhan, Xinyong Liu. 1-Hydroxypyrido[2,3-d]pyrimidin-2(1H)ones as novel selective HIV integrase inhibitors obtained via privileged substructure-based compound libraries. Bioorganic \& Medicinal Chemistry. 2017, vol. 25, iss.20, pp. 5779-5789. DOI: 10.1016/j.bmc.2017.09.006.

5. Emile J.Velthuisen, Brian A.Johns, Peter Gerondelis, Yan Chen, Ming Li, Ke Mou,
Wenwen Zhang, John W.Seal, Kendra E.Hightower, Sonia R.Miranda, Kevin Brown, Lisa Leesnitzer. Pyridopyrimidinone inhibitors of HIV-1 RNase H. European Journal of Medicinal Chemistry. 2014, vol. 83, pp. 609-616. DOI:10.1016/j.ejmech.2014.06.061.

6. Satyanarayana S., K. Praveen Kumar, P. Lakshmi Reddy, Narender R., Narasimhulu G., B.V. Subba Reddy. Microwave-assisted cyclocondensation: a rapid and solventfree synthesis of 3-benzyl-2H-pyrido[1,2a]pyrimidin-2-one derivatives. Tetrahedron Letters. 2013, vol.54, iss.36, pp. 4892-4895. DOI: 10.1016/j.tetlet.2013.06.138.

7. Hao Yan, Ying Ma, Yuanjun Sun, Chao Ma, Yuling Wang, Xiaoyu Ren, Guosheng Huang. Catalyst-free synthesis of alkyl 4oxo-4H-pyrido[1,2-a]pyrimidine-2carboxylate derivatives on water. Tetrahedron. 2014, vol.70, iss.17, pp. 27612765. DOI:10.1016/j.tet.2014.02.084.

8. Qun Cai, Mei-Cai Liu, Bi-Ming Mao, Xuan Xie, Feng-Cheng Jia, Yan-Ping Zhu, AnXin Wu. Direct one-pot synthesis of zolimidine pharmaceutical drug and imidazo[1,2-a]pyridine derivatives via $\mathrm{I}_{2} / \mathrm{CuO}$-promoted tandem strategy. Chinese Chemical Letters. 2015, vol. 26, iss.7, pp. 881-884.

DOI: 10.1016/j.cclet.2014.12.016.

9. Ullvi Bluhm, Jean-Luc Boucher, Uwe Buss, Bernd Clement, Friederike Friedrich, Ulrich Girreser, Dieter Heber, Thanh Lam, Michel Lepoivre, Mojgan RostaieGerylowa, Ulrich Wolschendorf. Synthesis and evaluation of pyrido[1,2-a]pyrimidines as inhibitors of nitric oxide synthases. 
European Journal of Medicinal Chemistry. 2009, vol. 44, iss.7, pp. 2877-2887.

DOI: 10.1016/j.ejmech.2008.12.007.

10. Zhengwang Chen, Yuelu Wen, Hao Ding, Guotian Luo, Min Ye, Liangxian Liu, Jun Xue. Silver-catalyzed highly efficient synthesis of pyrido[1,2-a]pyrimidin-4-ones from 2-aminopyridines and alkynoates. Tetrahedron Letters. 2017, vol.58, iss.1, p.13-16.

doi.org/10.1016/j.tetlet.2016.11.079.

11. Subhajit Mishra, Alakananda Hajra. Copper-catalyzed oxidative annulation between 2-aminopyridine and arylidenemalononitrile leading to 4-oxopyrido[1,2-a]pyrimidine-3-carbonitrile.

Tetrahedron Letters. 2015, vol. 56, iss. 41, pp. 5651-5655.

DOI: 10.1016/j.tetlet.2015.08.065.

12. Mehdi Adib, Hossine Yavari and Mehdi Mollahosseini. Efficient one-pot synthesis of 2-oxo-1,9a-dihydro-2H-pyrido-[1,2a]pyrimidine derivatives. Tetrahedron Letters. 2004, vol. 45, iss.8, pp. 18031805. DOI:10.1016/j.tetlet.2003.12.082.

\title{
BOZI ILIDENSIANOASETAMIDLORIN (VO YA ILIDENMALONONITRILLORIN) MALONONITRIL VӘ 1,3-DIAMINOPROPAN IL REAKSIYASININ TODQIQII
}

\author{
F.N. Nă̆ıyev \\ Bakı Dövlat Universiteti
}

AZ 1148 Bakl, Z.Xəlilov küç., 23; e-mail: farid.orgchemist@gmail.com

Ovazlanmiş ilidensianoasetamidlar (va ya ilidenmalononitrillarin) malononitril va 1,3-diaminopropan ila birmarhalali, üçkomponentli reaksiyası metanol mühitinda, otaq temperaturunda aparılmış va avazlanmiş yeni dihydropyridopirimidin töramalarinin amola galdiyi müayyən edilmişdir. Eyni reaksiya şəraitində piridilidensianoasetamid (vo ya piridilidenmalononitril), 2-xlor-5-nitrobenzylidensianoasetamid (vo ya 2xlor-5-nitrobenzylidenmalononitril), 2,6-dixlorbenzylidensianoasetamidin (vo ya 2,6-dixlorbenzylidenmalononitrilin)malononitril va 1,3-diaminpropan ila bir-marhalali, üçkomponentli reaksiyasindan uyğun avazlanmiş dihydropyridopirimidinlar deyil, tetrahydropyridopirimidin töramalarinin amala galmasi müəyyanlaşdirilmişdir. Bütün sintez edilmiş birlaşmalarin quruluşu NMR spektroskopiyası ila tasdiq edilmişdir.

Keywords: ilidensianoasetamidlor, piridilidensianoasetamid, malononitril, 1,3-diaminopropan, NMR

\section{ИССЛЕДОВАНИЕ ОДНОСТАДИЙНОЙ ТРЕХКОМПОНЕНТНОЙ РЕАКЦИИ НЕКОТОРЫХ ИЛИДЕНЦИАНОАЦЕТОАМИДОВ (ИЛИ ИЛИДЕНМАЛОНОНИТРИЛОВ), МАЛОНОНИТРИЛА И 1,3-ДИАМИНОПРОПАНА}

\author{
Ф.Н. Нагиев \\ Бакинский государственный университет \\ AZ 1148 Баку, ул. 3.Халилова, 23; e-mail: farid.orgchemist@gmail.com
}

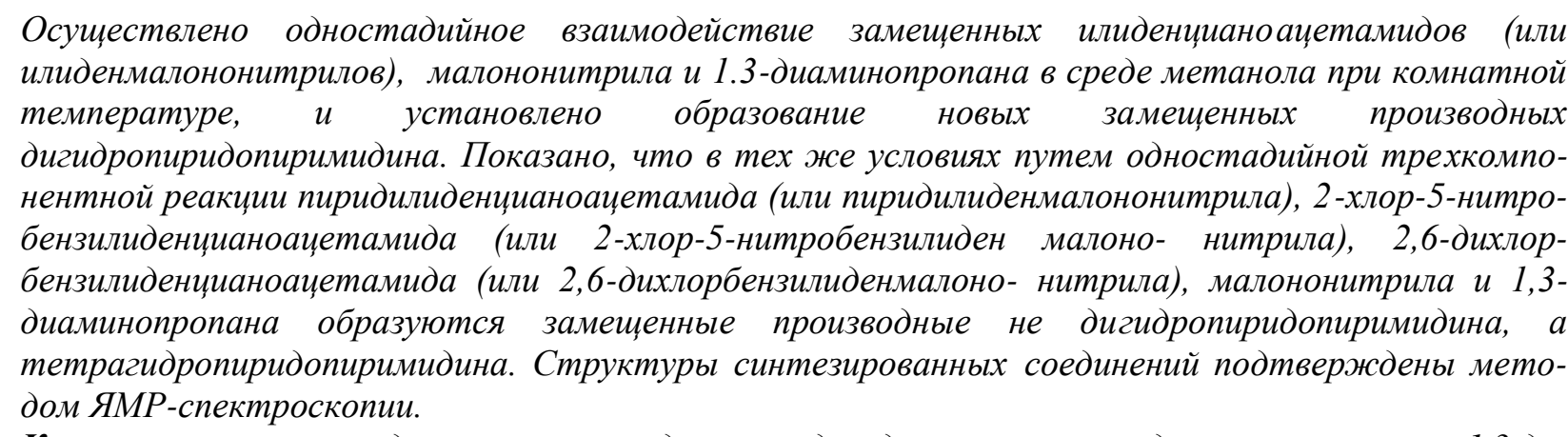
Ключевые слова: илидениианоаџетамиды, пиридилидениианоачетамид, малононитрил, 1,3-диаминопропан, ЯМР 